\title{
Criteria of boundedness and compactness of a class of matrix operators
}

\author{
Ryskul Oinarov ${ }^{*}$ and Zhanar Taspaganbetova
}

* Correspondence: o_ryskul@mail. ru

The L.N. Gumilyov Eurasian National University, Munaitpasov st., 5, 010008, Astana, Kazakhstan

\section{Abstract}

The aim of this article is to obtain criteria of boundedness and compactness for a wide class of matrix operators from one weighted $I_{p, v}$ space of sequences to another weighted $I_{q, u}$ space, in the case $1<p \leq q<\infty$. We introduce a general class of matrices. Then we establish necessary and sufficient conditions for the boundedness and compactness of the operators $\left(A^{+} f\right)_{i}:=\sum_{j=1}^{i} a_{i, j} f_{j}, i \geq 1$ and $\left(A^{-} f\right)_{j}:=\sum_{i=j}^{\infty} a_{i, j} f_{i}, j \geq 1$ corresponding to matrices in such classes by using the method of localization. Our classes are more general than those for which corresponding Hardy inequalities are known in the literature.

2010 Mathematics Subject Classification: 26D15; 47B37.

Keywords: inequalities, discrete Hardy-type inequalities, weights, matrix operators, boundedness, compactness

\section{Introduction}

We consider the problem of boundedness from the weighted $l_{p, v}$ space into the weighted $l_{q, u}$ space of the matrix operators

$$
\begin{aligned}
& \left(A^{+} f\right)_{i}:=\sum_{j=1}^{i} a_{i, j} f_{j}, \quad i \geq 1, \\
& \left(A^{-} f\right)_{j}:=\sum_{i=j}^{\infty} a_{i, j} f_{i,} \quad j \geq 1,
\end{aligned}
$$

which is equivalent to the validity of the following Hardy-type inequality

$$
\left\|A^{ \pm} f\right\|_{q, u} \leq C\|f\|_{p, v^{\prime}} \quad \forall f \in l_{p, v}
$$

where $C$ is a positive finite constant independent of $f$ and $\left(a_{i, j}\right)$ is a non-negative triangular matrix with entries $a_{i, j} \geq 0$ for $i \geq j \geq 1$ and $a_{i, j}=0$ for $i<j$.

Here and further $1<p, q<\infty, \frac{1}{p}+\frac{1}{p^{\prime}}=1$ and $u=\left\{u_{i}\right\}_{i=1}^{\infty}, v=\left\{v_{i}\right\}_{i=1}^{\infty}$ are positive sequences of real numbers. $l_{p, v}$ is the space of sequences $f=\left\{f_{i}\right\}_{i=1}^{\infty}$ of real numbers such that

\section{Springer}

(c) 2012 Oinarov and Taspaganbetova; licensee Springer. This is an Open Access article distributed under the terms of the Creative Commons Attribution License (http://creativecommons.org/licenses/by/2.0), which permits unrestricted use, distribution, and reproduction in any medium, provided the original work is properly cited. 


$$
\|f\|_{p, v}:=\left(\sum_{i=1}^{\infty}\left|v_{i} f_{i}\right|^{p}\right)^{\frac{1}{p}}<\infty, \quad 1<p<\infty .
$$

For $a_{i, j}=1, i \geq j \geq 1$, the operators (1), (2) coincide with the discrete Hardy operators of the forms $\left(A_{0}^{+} f\right)_{i}:=\sum_{j=1}^{i} f_{j},\left(A_{0}^{-} f\right)_{j}:=\sum_{i=j}^{\infty} f_{i}$, respectively. References about generalizations of the original forms of the discrete and continuous Hardy inequalities can be found in different books, see e.g., [1-3].

In $[4,5]$, necessary and sufficient conditions for the validity of (3) have been obtained for $1<p, q<\infty$ under the assumption that there exists $d \geq 1$ such that the inequalities

$$
\frac{1}{d}\left(a_{i, k}+a_{k, j}\right) \leq a_{i, j} \leq d\left(a_{i, k}+a_{k, j}\right), \quad i \geq k \geq j \geq 1
$$

hold.

A sequence $\left\{a_{i}\right\}_{i=1}^{\infty}$ is called almost non-decreasing (non-increasing), if there exists $c>0$ such that $c a_{i} \geq a_{k}\left(a_{k} \leq c a_{j}\right)$ for all $i \geq k \geq j \geq 1$.

In [6], estimate (3) has been studied under the assumption that there exist $d \geq 1$ and a sequence of positive numbers $\left\{\omega_{k}\right\}_{k=1}^{\infty}$, and a non-negative matrix $\left(b_{i, j}\right)$, where $b_{i, j}$ is almost non-decreasing in $i$ and almost non-increasing in $j$, such that the inequalities

$$
\frac{1}{d}\left(b_{i, k} \omega_{j}+a_{k, j}\right) \leq a_{i, j} \leq d\left(b_{i, k} \omega_{j}+a_{k, j}\right)
$$

hold for all $i \geq k \geq j \geq 1$.

In $[7,8]$, inequality (3) has been considered under the assumption that there exist $d \geq 1$, a sequence of positive numbers $\left\{\omega_{k}\right\}_{k=1}^{\infty}$, and a non-negative matrix $\left(b_{i, j}\right)$, whose entries $b_{i, j}$ are almost non-decreasing in $i$ and almost non-increasing in $j$ such that the inequalities

$$
\frac{1}{d}\left(a_{i, k}+b_{k, j} \omega_{i}\right) \leq a_{i, j} \leq d\left(a_{i, k}+b_{k, j} \omega_{i}\right)
$$

hold for all $i \geq k \geq j \geq 1$.

Conditions (5) and (6) include conditions (4), and complement each other.

In this article, we introduce a general class of matrices. We establish necessary and sufficient conditions for the boundedness and compactness of the operators (1) and (2), where the corresponding matrices belong to such classes. Such classes of matrices are wider than those which have been previously studied in the theory of discrete Hardy-type inequalities.

The content of the article is as follows. In Section 2, we introduce our classes of matrices and their properties. Moreover, in this section we give some auxiliary statements. Section 3 contains the main results. In Section 4, we prove the theorems, which give criteria of boundedness of the operators defined by (1) and (2). In Section 5, we obtain compactness criteria for the operators defined by (1) and (2). Then based on these statements, we prove our main theorems in Section 6. Moreover, in this section we show that one can imply our main results in order to obtain necessary and sufficient conditions for boundedness and compactness of the composition of operators.

Notation: If $M$ and $K$ are real valued functionals of sequences, then we understand that the symbol $M \ll K$ means that there exists $c>0$ such that $M \leq c K$, where $c$ is a 
constant which may depend only on parameters such as $p, q, r_{n}$ and $h_{n}$. If $M \ll K \ll$ $M$, then we write $M \approx K$.

\section{Preliminaries and notation}

For $n \geq 1$, we introduce the classes $\mathcal{O}_{n}^{+}$and $\mathcal{O}_{n}^{-}$of matrices $\left(a_{i, j}\right)$. We assume that $a_{i, j} \equiv a_{i, j}^{(n)}$ if $\left(a_{i, j}\right) \in \mathcal{O}_{n}^{+}$or $\left(a_{i, j}\right) \in \mathcal{O}_{n}^{-}$.

We define the classes $\mathcal{O}_{n}^{+}, n \geq 0$ by induction. Let $\left(a_{i, j}\right)$ be a matrix which is nonnegative and non-decreasing in the first index for all $i \geq j \geq 1$. By definition matrices of the type $a_{i, j}^{(0)}=\alpha_{j}, \forall i . \geq j \geq 1$ belong to the class $\mathcal{O}_{0}^{+}$. Let the classes $\mathcal{O}_{\gamma}^{+}, \gamma=0,1, \ldots$, $n-1, n \geq 1$ be defined. By definition, the matrix $\left(a_{i, j}\right) \equiv\left(a_{i, j}^{(n)}\right)$ belongs to the class $\mathcal{O}_{n}^{+}$ if and only if there exist matrices $\left(a_{i, j}^{(\gamma)}\right) \in \mathcal{O}_{\gamma}^{+}, \gamma=0,1, \ldots, n-1$ and a number $r_{n}>0$ such that

$$
a_{i, j}^{(n)} \leq r_{n} \sum_{\gamma=0}^{n} b_{i, k}^{n, \gamma} a_{k, j}^{(\gamma)}
$$

for all $i \geq k \geq j \geq 1$, where $b_{i, k}^{n, n} \equiv 1$ and

$$
b_{i, k}^{n, \gamma}=\inf _{1 \leq j \leq k} \frac{a_{i, j}^{(n)}}{a_{k, j}^{(\gamma)}}, \quad \gamma=0,1, \ldots, n-1 .
$$

From (8) it follows that entries of the matrices $\left(b_{i, k}^{n, \gamma}\right)$ do not decrease in the first index and do not increase in the second index. And (8) provides the validity of the following inequality

$$
\begin{aligned}
& a_{i, j}^{(n)} \geq b_{i, k}^{n, \gamma} a_{k, j}^{(\gamma)} \\
& i \geq k \geq j \geq 1, \gamma=0,1, \ldots, n, n=0,1, \ldots
\end{aligned}
$$

Then for $\left(a_{i, j}^{(n)}\right) \in \mathcal{O}_{n}^{+}$we have

$$
a_{i, j}^{(n)} \approx \sum_{\gamma=0}^{n} b_{i, k}^{n, \gamma} a_{k, j}^{(\gamma)}, \quad n \geq 0
$$

for all $i \geq k \geq j \geq 1$.

REMARK 1. It is easy to show that if for the matrix $\left(a_{i, j}^{(n)}\right), n \geq 0$ there exist matrices $\left(a_{i, j}^{(\gamma)}\right) \in \mathcal{O}_{\gamma}^{+}, \gamma=0,1, \ldots, n-1$, and matrices $\left(\tilde{b}_{i, k}^{n, \gamma}\right), \gamma=0,1, \ldots, n$ such that the equivalence (10) is valid for all $i \geq k \geq j \geq 1$, then $\left(a_{i, j}^{(n)}\right) \in \mathcal{O}_{n}^{+}$and $\tilde{b}_{i, k}^{n, \gamma} \approx b_{i, k}^{n, \gamma}$. Hence we may assume that the matrices $\left(b_{i, k}^{n, \gamma}\right)$ are arbitrary non-negative matrices which satisfy (10).

For the proof of our main results we also need the following inequality. Let $n \geq l \geq \gamma$. Then we have 


$$
b_{i, k}^{n, \gamma} \geq b_{i, s}^{n, l} \cdot b_{s, k}^{l, \gamma} \quad \forall i \geq s \geq k \geq 1
$$

Indeed, using (9), for $i \geq s \geq k \geq 1, n \geq l \geq \gamma$ we obtain

$$
b_{i, k}^{n, \gamma}=\inf _{1 \leq j \leq k} \frac{a_{i, j}^{(n)}}{a_{k, j}^{(\gamma)}} \geq b_{i, s}^{n, l} \cdot \inf _{1 \leq j \leq k} \frac{a_{s, j}^{(l)}}{a_{k, j}^{(\gamma)}}=b_{i, s}^{n, l} \cdot b_{s, k}^{l, \gamma} .
$$

As above, we introduce the classes $\mathcal{O}_{m}^{-}, m \geq 0$. Let $\left(a_{i, j}\right)$ be a matrix which is nonnegative and non-increasing in the second index for all $i \geq j \geq 1$. By definition a matrix $\left(a_{i, j}\right)=\left(a_{i, j}^{(0)}\right)$ belongs to the class $\mathcal{O}_{0}^{-}$if and only if it has the form $a_{i, j}^{(0)}=\beta_{i}$ for all $i \geq$ $j \geq 1$. Let the classes $\mathcal{O}_{\gamma}^{-}, \gamma=0,1, \ldots, m-1, m \geq 1$ be defined. A matrix $\left(a_{i, j}\right)=\left(a_{i, j}^{(m)}\right)$ belongs to the class $\mathcal{O}_{m}^{-}$if and only if there exist matrices $\left(a_{i, j}^{(\gamma)}\right) \in \mathcal{O}_{\gamma}^{-}, \gamma=0,1, \ldots, m$ 1 and a number $h_{m}>0$ such that

$$
a_{i, j}^{(m)} \leq h_{m} \sum_{\gamma=0}^{m} a_{i, k}^{(\gamma)} d_{k, j}^{\gamma, m},
$$

for all $i \geq k \geq j \geq 1$, where $d_{k, j}^{m, m} \equiv 1$ and

$$
d_{k, j}^{\gamma, m}=\inf _{k \leq i \leq \infty} \frac{a_{i, j}^{(m)}}{a_{i, k}^{(\gamma)}}, \quad \gamma=0,1, \ldots, m-1 .
$$

From the definition of the matrix $\left(d_{k, j}^{\gamma, m}\right), \gamma=0,1, \ldots, m-1, m=0,1, \ldots$, it is obvious that the entries of the matrix $\left(d_{k, j}^{\gamma, m}\right)$ do not decrease in the first index and do not increase in the second index and for $m \geq l \geq \gamma, k \geq s \geq j$ satisfy the following inequality

$$
d_{k, j}^{\gamma, m} \geq d_{k, s}^{\gamma, l} \cdot d_{s, j}^{l, m}
$$

From (13) it follows that for all $i \geq k \geq j \geq 1$

$$
a_{i, j}^{(m)} \geq a_{i, k}^{(\gamma)} d_{k, j}^{\gamma, m}, \quad \gamma=0,1, \ldots, m-1
$$

As in (10) every class $\mathcal{O}_{m}^{-}, m \geq 0$ of matrices $\left(a_{i, j}^{(m)}\right)$ is characterized by the following relation

$$
a_{i, j}^{(m)} \approx \sum_{\gamma=0}^{m} a_{i, k}^{(\gamma)} d_{k, j}^{\gamma, m},
$$

for all $i \geq k \geq j$, where $d_{k, j}^{\gamma, m}, \gamma=0,1, \ldots, m$ are defined by the formula (13).

REMARK 2. As mentioned before we may assume that the matrices $\left(d_{k, j}^{\gamma, m}\right), \gamma=0$, $1, \ldots, m, m \geq 0$ are arbitrary non-negative matrices which satisfy (16).

REMARK 3. By the definitions of the classes $\mathcal{O}_{n}^{ \pm}, n \geq 0$ we have $\mathcal{O}_{0}^{ \pm} \subset \mathcal{O}_{1}^{ \pm} \subset \cdots \subset \mathcal{O}_{n}^{ \pm} \subset \cdots$ 
In particular, the matrices of the classes $\mathcal{O}_{1}^{+}$and $\mathcal{O}_{1}^{-}$are characterized by the following relations, respectively,

$$
\begin{aligned}
& a_{i, j}^{(1)} \approx b_{i, k}^{1,0} a_{k, j}^{(0)}+a_{k, j}^{(1)} \quad \forall i \geq k \geq j \geq 1, \\
& a_{i, j}^{(1)} \approx a_{i, k}^{(1)}+a_{i, k}^{(0)} d_{k, j}^{0,1} \quad \forall i \geq k \geq j \geq 1 .
\end{aligned}
$$

It is easy to see that the class $\mathcal{O}_{1}^{+}$include the matrices, whose entries satisfy conditions (4) and (5). Also it should be noted that the matrices with conditions (4) and (6) belong to the class $\mathcal{O}_{1}^{-}$. This implies that the classes $\mathcal{O}_{n}^{+}, n \geq 1$ and $\mathcal{O}_{m}^{-}, m \geq 1$ are wider than the classes of matrices which have been used in this connection before.

The matrices of the classes $\mathcal{O}_{2}^{+}$and $\mathcal{O}_{2}^{-}$are described by the following relations, respectively,

$$
\begin{aligned}
& a_{i, j}^{(2)} \approx b_{i, k}^{2,0} a_{k, j}^{(0)}+b_{i, k}^{2,1} a_{k, j}^{(1)}+a_{k, j}^{(2)} \quad \forall i \geq k \geq j \geq 1, \\
& a_{i, j}^{(2)} \approx a_{i, k}^{(2)}+a_{i, k}^{(1)} d_{k, j}^{1,2}+a_{i, k}^{(0)} d_{k, j}^{0,2} \quad \forall i \geq k \geq j \geq 1 .
\end{aligned}
$$

Next, we show properties of the classes of matrices $\mathcal{O}_{n}^{+}$and $\mathcal{O}_{n}^{-}, n \geq 0$.

We set

$$
w_{i, k}=\sum_{j=k}^{i} a_{i, j} \sigma_{j, k} .
$$

Then we have the following

Lemma 2.1. Let $\left(a_{i, j}\right) \in \mathcal{O}_{n}^{+},\left(\sigma_{j, k}\right) \in \mathcal{O}_{m}^{+}$. Then $\left(w_{i, k}\right) \in \mathcal{O}_{m+n+1}^{+}$.

PROOF OF LEMMA 2.1. Since $\left(a_{i, j}\right) \in \mathcal{O}_{n}^{+}$, there exist matrices $\left(a_{i, j}^{(\gamma)}\right) \in \mathcal{O}_{\gamma}^{+}, \gamma=0$, $1, \ldots, n-1$, and matrices $\left(\delta_{i, l}^{n, \gamma}\right)$ such that

$$
a_{i, j} \equiv a_{i, j}^{(n)} \approx \sum_{\gamma=0}^{n} \delta_{i, l}^{n, \gamma} a_{l, k}^{(\gamma)}, \quad n=0,1, \ldots, \quad \delta_{i, l}^{n, n} \equiv 1
$$

for all $i \geq l \geq k \geq 1$.

Since $\left(\sigma_{j, k}\right) \in \mathcal{O}_{m}^{+}$, there exist matrices $\left(\sigma_{j, k}^{(\mu)}\right) \in \mathcal{O}_{\mu}^{+}, \mu=0,1, \ldots, m-1$, and matrices $\left(e_{k, l}^{m, \mu}\right)$ such that

$$
\sigma_{j, k} \equiv \sigma_{j, k}^{(m)} \approx \sum_{\mu=0}^{m} e_{j, l}^{m, \mu} \sigma_{l, k}^{(\mu)}, \quad m=0,1, \ldots, \quad e_{j, l}^{m, m} \equiv 1
$$

for all $j \geq l \geq k \geq 1$.

We set

$$
w_{i, k} \equiv w_{i, k}^{n, m}=\sum_{j=k}^{i} a_{i, j}^{(n)} \sigma_{j, k}^{(m)} .
$$

First, we consider the case when $m \geq 0, n=0$. In this case $a_{i, j}^{(0)}=\alpha_{j}, \forall i \geq j \geq 1$. For $\forall i \geq l \geq k$ we obtain 


$$
w_{i, k}^{o, m}=\sum_{j=k}^{i} \alpha_{j} \sigma_{j, k}^{(m)} \approx \sum_{j=k}^{l} \alpha_{j} \sigma_{j, k}^{(m)}+\sum_{j=l}^{i} \alpha_{j} \sigma_{j, k}^{(m)} \approx w_{l, k}^{o, m}+\sum_{\mu=0}^{m} \sigma_{l, k}^{(\mu)} \sum_{j=l}^{i} \alpha_{j} e_{j, l}^{m, \mu}=w_{l, k}^{o, m}+\sum_{\mu=0}^{m} \tilde{e}_{i, l}^{m+1, \mu} \sigma_{l, k}^{(\mu)},
$$

where $\tilde{e}_{i, l}^{m+1, \mu}=\sum_{j=l}^{i} \alpha_{j} e_{j, l}^{m, \mu}, \mu=0,1, \ldots, m$. Suppose that $\tilde{e}_{i, l}^{m+1, m+1} \equiv 1$. Since $\left(\sigma_{l, k}^{(\mu)}\right) \in \mathcal{O}_{\mu}^{+}, \mu=0,1, \ldots, m$, by definition we easily see that $w_{i, k}^{o, m} \in \mathcal{O}_{m+1}^{+}$. By induction, we assume that for $n=0,1, \ldots, r-1, r \geq 1\left(w_{i, k}^{n, m}\right)$ belongs to the class $\mathcal{O}_{n+m+1}^{+}$. For $i \geq l \geq k$ we have

$$
\begin{aligned}
w_{i, k}^{r, m} & =\sum_{j=k}^{i} a_{i, j}^{(r)} \sigma_{j, k}^{(m)} \approx \sum_{j=k}^{l} a_{i, j}^{(r)} \sigma_{j, k}^{(m)}+\sum_{j=l}^{i} a_{i, j}^{(r)} \sigma_{j, k}^{(m)} \\
& \approx \sum_{j=k}^{l}\left(\sum_{\gamma=0}^{r} \delta_{i, l}^{r, \gamma} a_{l, j}^{(\gamma)}\right) \sigma_{j, k}^{(m)}+\sum_{j=l}^{i} a_{i, j}^{(r)}\left(\sum_{\mu=0}^{m} e_{j, l}^{m, \mu} \sigma_{l, k}^{(\mu)}\right) \\
& =\sum_{\gamma=0}^{r} \delta_{i, l}^{r, \gamma} \sum_{j=k}^{l} a_{l, j}^{(\gamma)} \sigma_{j, k}^{(m)}+\sum_{\mu=0}^{m} \sigma_{l, k}^{(\mu)} \sum_{j=l}^{i} a_{i, j}^{(r)} e_{j, l}^{m, \mu} \\
& =\sum_{j=k}^{l} a_{l, j}^{(r)} \sigma_{j, k}^{(m)}+\sum_{\gamma=0}^{r-1} \delta_{i, l}^{r, \gamma} \sum_{j=k}^{l} a_{l, j}^{(\gamma)} \sigma_{j, k}^{(m)}+\sum_{\mu=0}^{m} \sigma_{l, k}^{(\mu)} \sum_{j=l}^{i} a_{i, j}^{(r)} e_{j, l}^{m, \mu} \\
& =w_{l, k}^{r, m}+\sum_{\gamma=0}^{r-1} \delta_{i, l}^{r, \gamma} \tilde{\sigma}_{l, k}^{(\gamma+m+1)}+\sum_{\mu=0}^{m} \tilde{e}_{i, l}^{m+1, \mu} \sigma_{l, k}^{(\mu)},
\end{aligned}
$$

where $\tilde{\sigma}_{l, k}^{(\gamma+m+1)} \equiv \sum_{j=k}^{l} a_{l, j}^{(\gamma)} \sigma_{j, k}^{(m)}, \gamma=0, \ldots, r-1$ and $\tilde{e}_{i, l}^{m+1, \mu} \equiv \sum_{j=l}^{i} a_{i, j}^{(r)} e_{j, l}^{m, \mu} \mu=0, \ldots, m$. We denote $\gamma+m+1$ by $\mu$. Then we have

$$
\begin{aligned}
w_{i, k}^{r, m} & \approx w_{l, k}^{r, m}+\sum_{\mu=m+1}^{r+m} \delta_{i, l}^{r, \mu-m-1} \tilde{\sigma}_{l, k}^{(\mu)}+\sum_{\mu=0}^{m} \tilde{e}_{i, l}^{m+1, \mu} \sigma_{l, k}^{(\mu)} \\
& =w_{l, k}^{r, m}+\sum_{\mu=0}^{r+m} \tilde{\delta}_{i, l}^{r+m, \mu} \tilde{\sigma}_{l, k}^{(\mu)}
\end{aligned}
$$

where

$$
\tilde{\delta}_{i, l}^{r+m, \mu}=\left\{\begin{array}{l}
\tilde{e}_{i, l}^{m+1, \mu}, 0 \leq \mu \leq m \\
\delta_{i, l}^{r, \mu-m-1}, m+1 \leq \mu \leq r+m
\end{array}\right.
$$

and

$$
\tilde{\sigma}_{l, k}^{(\mu)}=\left\{\begin{array}{l}
\sigma_{l, k}^{(\mu)}, 0 \leq \mu \leq m \\
\tilde{\sigma}_{l, k}^{(\mu)}, m+1 \leq \mu \leq r+m
\end{array}\right.
$$

Since $\tilde{\sigma}_{l, k}^{(\mu)} \in \mathcal{O}_{\mu}^{+}, \mu=0,1, \ldots, r+m$ we obtain that $w_{i, k}^{r, m} \in \mathcal{O}_{r+m+1}^{+}$. The proof is complete. 
Now we set

$$
\varphi_{k, j}=\sum_{i=j}^{k} \sigma_{k, i} a_{i, j} .
$$

Then we have the following lemma.

Lemma 2.2. Let $\left(a_{i, j}\right) \in \mathcal{O}_{n}^{-},\left(\sigma_{k, i}\right) \in \mathcal{O}_{m}^{-}$. Then $\left(\varphi_{k, j}\right) \in \mathcal{O}_{m+n+1}^{-}$.

Lemma 2.2 can be proved in the same way as Lemma 2.1.

For the proof of our main theorem we will need the following well-known result for the discrete weighted Hardy inequality (see $[1,9]$ ) and the criteria of precompactness of sets in $l_{p}$ (see [10, p. 32]).

Theorem A. Let $1<p \leq q<\infty$. Then the inequality

$$
\left(\sum_{j=1}^{\infty}\left(\sum_{i=j}^{\infty} \omega_{i} f_{i}\right)^{q} u_{j}^{q}\right)^{\frac{1}{q}} \leq C\left(\sum_{i=1}^{\infty}\left|v_{i} f_{i}\right|^{p}\right)^{\frac{1}{p}}, \quad 0 \leq f \in l_{p, v}
$$

holds if and only if

$$
H:=\sup _{n \geq 1}\left(\sum_{j=1}^{n} u_{j}^{q}\right)^{\frac{1}{q}}\left(\sum_{i=n}^{\infty} \omega_{i}^{p^{\prime}} v_{i}^{-p^{\prime}}\right)^{\frac{1}{p^{\prime}}}<\infty .
$$

Moreover, $H \approx C$, where $C$ is the best constant in (17).

Theorem B. Let $T$ be a set in $l_{p}, 1 \leq p<\infty$. The set $T$ is compact if and only if $T$ is bounded and for all $\varepsilon>0$ there exists $N=N(\varepsilon)$ such that for all $x=\left\{x_{i}\right\}_{i=1}^{\infty} \in T$ the inequality

$$
\sum_{i=N}^{\infty}\left|x_{i}\right|^{p}<\varepsilon
$$

holds.

\section{Main results}

We define

$$
\begin{aligned}
& \left(\mathcal{B}_{p, q}^{+}\right)_{k}=\left(\sum_{j=1}^{k} v_{j}^{-p^{\prime}}\left(\sum_{i=k}^{\infty} a_{i, j}^{q} u_{i}^{q}\right)^{\frac{p^{\prime}}{q}}\right)^{\frac{1}{p^{\prime}}}, \\
& \left(\mathcal{B}_{p, q}^{-}\right)_{k}=\left(\sum_{i=k}^{\infty} u_{i}^{q}\left(\sum_{j=1}^{k} a_{i, j}^{p^{\prime}} v_{j}^{-p^{\prime}}\right)^{\frac{q}{p^{\prime}}}\right)^{\frac{1}{q}}, \\
& \left(\mathcal{A}_{p, q}^{+}\right)_{k}=\left(\sum_{j=1}^{k} u_{j}^{q}\left(\sum_{i=k}^{\infty} a_{i, j}^{p^{\prime}} v_{i}^{-p^{\prime}}\right)^{\frac{q}{p^{\prime}}}\right)^{\frac{1}{q}}, \\
& \left(\mathcal{A}_{p, q}^{-}\right)_{k}=\left(\sum_{i=k}^{\infty} v_{i}^{-p^{\prime}}\left(\sum_{j=1}^{k} a_{i, j}^{q} u_{j}^{q}\right)^{\frac{p^{\prime}}{q}}\right)^{\frac{1}{p^{\prime}}} .
\end{aligned}
$$


We set $\mathcal{B}^{+}=\sup _{k \geq 1}\left(\mathcal{B}_{p, q}^{+}\right)_{k}, \mathcal{B}^{-}=\sup _{k \geq 1}\left(\mathcal{B}_{p, q}^{-}\right)_{k}, \mathcal{A}^{+}=\sup _{k \geq 1}\left(\mathcal{A}_{p, q}^{+}\right)_{k}$ and $\mathcal{A}^{-}=\sup _{k \geq 1}\left(\mathcal{A}_{p, q}^{-}\right)_{k}$.

Theorem 3.1. Suppose that $1<p \leq q<\infty$. Let the matrix $\left(a_{i, j}\right)$ in (1) belong to the class $\mathcal{O}_{m}^{+} \cup \mathcal{O}_{m}^{-}, m \geq 0$. Let $A^{+}$be the operator defined in (1). Then the following statements hold:

(i) $A^{+}$is bounded from $l_{p, v}$ into $l_{q, u}$ if and only if one of the conditions $\mathcal{B}^{+}<\infty$ and $\mathcal{B}^{-}<\infty$ holds. Moreover $\mathcal{B}^{+} \approx \mathcal{B}^{-} \approx C$, where $C$ is the best constant in (3).

(ii) $A^{+}$is compact from $l_{p, v}$ into $l_{q, u}$ if and only if one of the conditions $\lim _{k \rightarrow \infty}\left(\mathcal{B}_{p, q}^{+}\right)_{k}=0$ and $\lim _{k \rightarrow \infty}\left(\mathcal{B}_{p, q}^{-}\right)_{k}=0$ holds.

Theorem 3.2. Suppose that $1<p \leq q<\infty$. Let the matrix $\left(a_{i, j}\right)$ in (2) belong to the class $\mathcal{O}_{m}^{+} \cup \mathcal{O}_{m}^{-}, m \geq 0$. Let $A^{-}$be the operator defined in (2). Then the following statements hold:

(j) $A^{-}$is bounded from $l_{p, v}$ into $l_{q, u}$ if and only if one of the conditions $\mathcal{A}^{+}<\infty$ and $\mathcal{A}^{-}<\infty$ holds. Moreover $\mathcal{A}^{+} \approx \mathcal{A}^{-} \approx C$, where $C$ is the best constant in (3).

(ji) $A^{-}$is compact from $l_{p, v}$ into $l_{q, u}$ if and only if one of the conditions $\lim _{k \rightarrow \infty}\left(\mathcal{A}_{p, q}^{+}\right)_{k}=0$ and $\lim _{k \rightarrow \infty}\left(\mathcal{A}_{p, q}^{-}\right)_{k}=0$ holds.

Before proving our main theorems we establish the boundedness and compactness of the operators $\left(A^{+} f\right)_{i}:=\sum_{j=1}^{i} a_{i, j} f_{j}, i \geq 1$ and $\left(A^{-} f\right)_{j}:=\sum_{i=j}^{\infty} a_{i, j} f_{i,} \quad j \geq 1$ from the weighted $l_{p, v}$ space into the weighted $l_{q, u}$ space in particular cases.

\section{Boundedness of the matrix operators}

Theorem 4.1. Let $1<p \leq q<\infty$. Let the matrix $\left(a_{i, j}\right)$ in (1) belong to the class $\mathcal{O}_{n}^{+}, n \geq 0$. Then the estimate (3) for the operator defined by (1) holds if and only if one of the conditions $\mathcal{B}^{+}<\infty$ and $\mathcal{B}^{-}<\infty$ holds. Moreover $\mathcal{B}^{+} \approx \mathcal{B}^{-} \approx C$, where $C$ is the best constant in (3).

Theorem 4.2. Let $1<p \leq q<\infty$. Let the matrix $\left(a_{i, j}\right)$ in (2) belong to the class $\mathcal{O}_{m}^{-}, m \geq 0$. Then the estimate (3) for the operator defined by (2) holds if and only if one of the conditions $\mathcal{A}^{+}<\infty$ and $\mathcal{A}^{-}<\infty$ holds. Moreover $\mathcal{A}^{+} \approx \mathcal{A}^{-} \approx C$, where $C$ is the best constant in (3).

Here we present only the proof of Theorem 4.2, since the proof of Theorem 4.1 is very similar.

For the proof of Theorem 4.2 we need the following.

Lemma 4.1. Let the matrix of (2) belongs to the class $\mathcal{O}_{m}^{-}, m \geq 0$. Then we have the following equivalence

$$
\left(\mathcal{A}_{p, q}^{+}\right)_{k} \approx\left(\mathcal{A}_{m}\right)_{k} \equiv \max _{0 \leq \gamma \leq m}\left(\mathcal{A}_{\gamma, m}\right)_{k} \approx\left(\mathcal{A}_{p, q}^{-}\right)_{k}
$$

where

$$
\left(\mathcal{A}_{\gamma, m}\right)_{k}=\left(\sum_{j=1}^{k}\left(d_{k, j}^{\gamma, m}\right)^{q} u_{j}^{q}\right)^{\frac{1}{q}}\left(\sum_{i=k}^{\infty}\left(a_{i, k}^{(\gamma)}\right)^{p^{\prime}} v_{i}^{-p^{\prime}}\right)^{\frac{1}{p^{\prime}}} .
$$


By (18) it follows that

$$
\mathcal{A}^{+} \approx \mathcal{A}_{m}=\sup _{k \geq 1}\left(\mathcal{A}_{m}\right)_{k} \approx \mathcal{A}^{-}, \quad \forall m \geq 0 .
$$

Indeed, this equivalence easily follows from (16).

PROOF OF THEOREM 4.2. Necessity. Suppose that the matrix of the operator (2) belongs to the class $\mathcal{O}_{m}^{-}, m \geq 0$ and (3) holds.

For $k>1$ we assume that $g=\left\{g_{i}\right\}_{i=1}^{\infty}: \quad g_{i}=\left\{\begin{array}{l}u_{i}, 1 \leq i \leq k \\ 0, i>k .\end{array}\right.$

It is known that inequality (3) holds if and only if the following dual inequality

$$
\left\|A^{*} g\right\|\left\|_{p^{\prime}, v^{-1}} \leq C\right\| g \|_{q^{\prime}, u^{-1}}, \quad g \in l_{q^{\prime}, u^{-1}}
$$

holds for the conjugate operator $A$ *, which coincides with operator defined by (1). Moreover, the best constants in (3) and (20) coincide (see e.g., [3]).

Hence applying $g=\left\{g_{i}\right\}_{i=1}^{\infty}$ in (20) and using (15) we obtain

$$
\begin{aligned}
C k^{\frac{1}{q^{\prime}}} & \geq\left\|A^{*} g\right\|_{p^{\prime}, v^{-1}} \geq\left(\sum_{i=k}^{\infty}\left(\sum_{j=1}^{k} a_{i, j}^{(m)} u_{j}\right)^{p^{\prime}} v_{i}^{-p^{\prime}}\right)^{\frac{1}{p^{\prime}}} \\
& \gg\left(\sum_{i=k}^{\infty}\left(a_{i, k}^{(\gamma)}\right)^{p^{\prime}} v_{i}^{-p^{\prime}}\right)^{\frac{1}{p^{\prime}}}\left(\sum_{j=1}^{k} d_{k, j}^{\gamma, m} u_{j}\right), \quad \gamma=0,1, \ldots, m .
\end{aligned}
$$

Therefore $\left\{a_{i, k}^{(\gamma)_{i=1}^{\infty}} \in l_{p^{\prime}, v^{-1}}\right.$.

Now for $1 \leq r<M<\infty$, we assume that $f=\left\{f_{s}\right\}_{s=1}^{\infty}$, where

$$
f_{s}= \begin{cases}\left(a_{s, r}^{(\gamma)}\right)^{p^{\prime}-1} v_{s}^{-p^{\prime}}, & r \leq s \leq M \\ 0, & s<r \quad \text { or } \quad s>M .\end{cases}
$$

Applying $f$ to inequality (3) and using (15) we find that

$$
\begin{aligned}
C\left(\sum_{s=r}^{M}\left(a_{s, r}^{(\gamma)}\right)^{p^{\prime}} v_{s}^{-p^{\prime}}\right)^{\frac{1}{p}} & \geq\left\|A^{-} f\right\|_{q, u}=\left(\sum_{j=1}^{\infty}\left(\sum_{s=j}^{\infty} a_{s, j}^{(m)} f_{s}\right)^{q} u_{j}^{q}\right)^{\frac{1}{q}} \\
& \gg\left(\sum_{j=1}^{r}\left(d_{r, j}^{\gamma, m}\right)^{q} u_{j}^{q}\right)^{\frac{1}{q}}\left(\sum_{s=r}^{M}\left(a_{s, r}^{(\gamma)}\right)^{p^{\prime}} v_{s}^{-p^{\prime}}\right),
\end{aligned}
$$

which implies

$$
C \gg\left(\sum_{j=1}^{r}\left(d_{r, j}^{\gamma, m}\right)^{q} u_{j}^{q}\right)^{\frac{1}{q}}\left(\sum_{s=r}^{M}\left(a_{s, r}^{(\gamma)}\right)^{p^{\prime}} v_{s}^{-p^{\prime}}\right)^{\frac{1}{p^{\prime}}} .
$$

Since inequality (21) holds for all $\gamma=0,1, \ldots, m$ and $r \geq 1$ is arbitrary, passing to the limit as $M \rightarrow \infty$ we have 


$$
\sup _{k \geq 1}\left(\mathcal{A}_{m}\right)_{k} \ll C
$$

By using Lemma 4.1 we obtain

$$
\mathcal{A}^{+} \approx \mathcal{A}^{-} \ll C
$$

The proof of the necessity is complete.

Sufficiency. Let the matrix $\left(a_{i, j}\right)$ of the operator (2) belong to the class $\mathcal{O}_{m}^{-}, m \geq 0$. Let $0 \leq f \in l_{p, v}$ and at least one of the conditions $\mathcal{A}^{+}<\infty$ and $\mathcal{A}^{-}<\infty$ hold. Assume that $m=0$. By the definition of $\mathcal{O}_{0}^{-}$, the matrix of the operator (2) has the form $a_{i, j}^{(0)}=\beta_{i} \forall i \geq j \geq 1$. Then the estimate (3) coincides with the estimate (17) and the operator (2) is the matrix operator $A_{0}^{-}$. Hence from Theorem $\mathrm{A}$ it follows that

$$
\left\|A_{0}^{-} f\right\|_{q, u} \ll \mathcal{A}_{0}\|f\|_{p, v}, \quad \forall f \in l_{p, v}
$$

Based on Lemma 4.1 it follows that the inequality (3) holds for $m=0$ and for the best constant in (3) the following estimate is valid

$$
C \ll \mathcal{A}^{+} \approx \mathcal{A}^{-}
$$

Now we assume that the inequality (3) holds for $m=0,1, \ldots, n-1, n \geq 1$ and for the best constant in (3) the estimate (24) is valid. We consider the inequality

$$
\left\|A_{m}^{-} f\right\|_{q, u} \ll \mathcal{A}_{m}\|f\|_{p, v}, \quad \forall f \in l_{p, v}
$$

where $A_{m}^{-}$is given by (2) with the matrix $\left(a_{i, j}^{(m)}\right) \in \mathcal{O}_{m}^{-}$.

Now our aim is to show that the inequality (25) holds for $m=n$ with the estimate (24).

Let $h \equiv h_{n}$, where $h_{n}$ is the constant in (12) with $m=n$. For all $j \geq 1$ we define the following set:

$$
T_{j}=\left\{k \in \mathbb{Z}:(h+1)^{-k} \leq\left(A_{n}^{-} f\right)_{j}\right\},
$$

where $\mathbb{Z}$ is the set of integers. We assume that $k_{j}=\inf T_{j}$, if $T_{j} \neq \varnothing$ and $k_{j}=\infty$, if $T_{j}$ $=\varnothing$. In order to avoid trivial cases we directly suppose that $\left(A_{n}^{-} f\right)_{1} \neq 0$. Since $a_{i, j}^{(n)}$ is non-increasing in $j$, we have $k_{j} \leq k_{j+1}$. If $k_{j}<\infty$, then

$$
(h+1)^{-k_{j}} \leq\left(A_{n}^{-} f\right)_{j}<(h+1)^{-\left(k_{j}-1\right)}, \quad j \geq 1 .
$$

Let $m_{1}=0, k_{1}=k_{m_{1}+1}$ and $M_{1}=\left\{j\left\lfloor\mathbb{N}: k_{j}=k_{1}=k_{m_{1}+1}\right\}\right.$, where $\mathbb{N}$ is the set of natural numbers. Suppose that $m_{2}$ is such that sup $M_{1}=m_{2}$. Obviously $m_{2}>m_{1}$ and if the set $M_{1}$ is bounded from above, then $m_{2}<\infty$ and $m_{2}=\max M_{1}$. We now define the numbers $0=m_{1}<m_{2}<\cdots<m_{s}<\infty, s \geq 1$ by induction. To define $m_{s+1}$ we assume that $m_{s+1}=\sup M_{s}$, where $M_{s}=\left\{j \in \mathbb{N}: k_{j}=k_{m_{s+1}}\right\}$.

Let $N_{0}=\left\{s \in \mathbb{N}: m_{s}<\infty\right\}$. Further, we assume that $k_{m_{s+1}}=n_{s+1}, s \in N_{0}$. From the definition of $m_{s}$ and from (26) it follows that, for $s \in N_{0}$,

$$
(h+1)^{-n_{s+1}} \leq\left(A_{n}^{-} f\right)_{j}<(h+1)^{-n_{s+1}+1}, \quad m_{s}+1 \leq j \leq m_{s+1}
$$


and

$$
\mathbb{N}=\bigcup_{s \in N_{0}}\left[m_{s}+1, m_{s+1}\right], \quad \text { where }\left[m_{s}+1, m_{s+1}\right] \cap\left[m_{l}+1, m_{l+1}\right]=\emptyset, s \neq l .
$$

Therefore for $0 \leq f \in l_{p, v}$ the left-hand side of (3) has the following form

$$
\left\|A_{n}^{-} f\right\|_{q, u}^{q}=\sum_{s \in N_{0}} \sum_{j=m_{s}+1}^{m_{s+1}}\left(A_{n}^{-} f\right)_{j}^{q} u_{j}^{q}
$$

We assume that $\sum_{j=m_{s}+1}^{m_{s+1}}=0$, if $m_{s}=\infty$.

There are two possible cases: $N_{0}=\mathbb{N}$ and $N_{0} \neq \mathbb{N}$.

1. If $N_{0}=\mathbb{N}$, then we estimate (28) in the following way.

Clearly inequalities $n_{s+1}<n_{s+2}<n_{s+3}$ imply that $-n_{s+3}+1 \leq-n_{s+1}-1$ for all $s \in \mathbb{N}$. Hence, (27), (16) imply that

$$
\begin{aligned}
(h+1)^{-n_{s+1}-1} & =(h+1)^{-n_{s+1}}-h(h+1)^{-n_{s+1}-1} \\
& \leq(h+1)^{-n_{s+1}}-h(h+1)^{-n_{s+3}+1}<\left(A_{n}^{-} f\right)_{m_{s+1}}-h\left(A_{n}^{-} f\right)_{m_{s+3}} \\
& =\sum_{i=m_{s+1}}^{\infty} a_{i, m_{s+1}}^{(n)} f_{i}-h \sum_{i=m_{s+3}}^{\infty} a_{i, m_{s+3}}^{(n)} f_{i} \\
& \leq \sum_{i=m_{s+1}}^{m_{s+3}} a_{i, m_{s+1}}^{(n)} f_{i}+\sum_{i=m_{s+3}}^{\infty}\left[a_{i, m_{s+1}}^{(n)}-h a_{i, m_{s+3}}^{(n)}\right] f_{i} \\
& \leq \sum_{i=m_{s+1}}^{m_{s+3}} a_{i, m_{s+1}}^{(n)} f_{i}+\sum_{i=m_{s+3}}^{\infty}\left[h \sum_{\gamma=0}^{n} a_{i, m_{s+3}}^{(\gamma)} d_{m_{s+3}, m_{s+1}}^{\gamma, n}-h a_{i, m_{s+3}}^{(n)}\right] f_{i} \\
& =\sum_{i=m_{s+1}}^{m_{s+3}} a_{i, m_{s+1}}^{(n)} f_{i}+h \sum_{i=m_{s+3}}^{\infty} \sum_{\gamma=0}^{n-1} a_{i, m_{s+3}}^{(\gamma)} d_{m_{s+3}, m_{s+1}}^{\gamma, n} f_{i} .
\end{aligned}
$$

Now, by using (27) and (29), we can estimate (28) in the following way.

$$
\begin{gathered}
\sum_{s \in N} \sum_{j=m_{s}+1}^{m_{s+1}}\left(A_{n}^{-} f\right)_{j}^{q} u_{j}^{q}<\sum_{s \in N} \sum_{j=m_{s}+1}^{m_{s+1}}(h+1)^{\left(-n_{s+1}+1\right) q} u_{j}^{q} \\
=(h+1)^{2 q} \sum_{s \in N}(h+1)^{\left(-n_{s+1}-1\right) q} \sum_{j=m_{s}+1}^{m_{s+1}} u_{j}^{q} \\
\ll \sum_{s \in N}\left(\sum_{i=m_{s+1}}^{m_{s+3}} a_{i, m_{s+1}}^{(n)} f_{i}+h \sum_{\gamma=0}^{n-1} \sum_{i=m_{s+3}}^{\infty} a_{i, m_{s+3}}^{(\gamma)} d_{m_{s+3}, m_{s+1}}^{\gamma, n} f^{q}\right)_{j=m_{s}+1}^{m_{s+1}} u_{j}^{q} \\
\ll \sum_{s \in N}\left(\sum_{i=m_{s+1}}^{m_{s+3}} a_{i, m_{s+1}}^{(n)} f_{i}\right)^{q} \sum_{j=m_{s}+1}^{m_{s+1}} u_{j}^{q} \\
+\sum_{\gamma=0}^{n-1} \sum_{s \in N}\left(d_{m_{s+3}, m_{s+1}}^{\gamma, n}\right)^{q}\left(\sum_{i=m_{s+3}}^{\infty} a_{i, m_{s+3}}^{(\gamma)} f_{i}\right)^{q} \sum_{j=m_{s}+1}^{m_{s+1}} u_{j}^{q}:=I_{n}+\sum_{\gamma=0}^{n-1} I_{\gamma}
\end{gathered}
$$

where

$$
I_{n}=\sum_{s \in N}\left(\sum_{i=m_{s+1}}^{m_{s+3}} a_{i, m_{s+1}}^{(n)} f_{i}\right)^{q} \sum_{j=m_{s}+1}^{m_{s+1}} u_{j}^{q}
$$


and

$$
I_{\gamma}=\sum_{s \in N}\left(d_{m_{s+3}, m_{s+1}}^{\gamma, n}\right)^{q}\left(\sum_{i=m_{s+3}}^{\infty} a_{i, m_{s+3}}^{(\gamma)} f_{i}\right)^{q} \sum_{j=m_{s}+1}^{m_{s+1}} u_{j}^{q}, \quad 0 \leq \gamma \leq n-1 .
$$

To estimate $I_{n}$ we apply Hölder's and Jensen's inequalities and find that

$$
\begin{gathered}
I_{n} \leq \sum_{s \in N}\left(\sum_{i=m_{s+1}}^{m_{s+3}}\left(a_{i, m_{s+1}}^{(n)}\right)^{p^{\prime}} v_{i}^{-p^{\prime}}\right)^{\frac{q}{p^{\prime}}} \sum_{j=m_{s}+1}^{m_{s+1}} u_{j}^{q}\left(\sum_{i=m_{s+1}}^{m_{s+3}}\left|f_{i} v_{i}\right|^{p}\right)^{\frac{q}{p}} \\
\leq\left[\sup _{k \geq 1}\left(\sum_{j=1}^{k} u_{j}^{q}\right)^{\frac{1}{q}}\left(\sum_{i=k}^{\infty}\left(a_{i, k}^{(n)}\right)^{p^{\prime}} v_{i}^{-p^{\prime}}\right)^{\frac{1}{p^{\prime}}}\right]^{q} \sum_{s \in N}\left(\sum_{j=m_{s+1}}^{m_{s+3}}\left|f_{i} v_{i}\right|^{p}\right)^{\frac{q}{p}} \\
\leq\left[\sup _{k \geq 1}\left(\sum_{j=1}^{k}\left(d_{k, j}^{n, n}\right)^{q} u_{j}^{q}\right)^{\frac{1}{q}}\left(\sum_{i=k}^{\infty}\left(a_{i, k}^{(n)}\right)^{p^{\prime}} v_{i}^{-p^{\prime}}\right)^{\frac{1}{p^{\prime}}}\right]^{q}\left(\sum_{s \in N} \sum_{i=m_{s+1}}^{m_{s+3}}\left|f_{i} v_{i}\right|^{p}\right)^{\frac{q}{p}} \ll \mathcal{A}_{n}^{q}|| f||_{p, v}^{q} .
\end{gathered}
$$

We introduce the sequence $\left\{\Delta_{j}\right\}_{j=1}^{\infty}$ defined by $\Delta_{j}=\left(d_{m_{s+3}, m_{s+1}}^{\gamma, n}\right)^{q} \sum_{j=m_{s}+1}^{m_{s+1}} u_{j}^{q}, j=m_{s+3}$ and $\Delta_{j}=0, j \neq m_{s+3}, s \in N$. Hence, we can rewrite $I_{\gamma} \gamma=0, \ldots, n-1$ in the following form:

$$
\begin{gathered}
I_{\gamma}=\sum_{s \in N}\left(\sum_{i=m_{s+3}}^{\infty} a_{i, m_{s+3}}^{(\gamma)} f_{i}\right)^{q}\left(d_{m_{s+3}, m_{s+1}}^{\gamma, n}\right)^{q} \sum_{i=m_{s}+1}^{m_{s+1}} u_{i}^{q} \\
=\sum_{j=1}^{\infty}\left(\sum_{i=j}^{\infty} a_{i, j}^{(\gamma)} f_{i}\right)^{q} \Delta_{j} .
\end{gathered}
$$

By the assumptions on $a_{i, j}^{(\gamma)}, \gamma=0, \ldots, n-1, i \geq j \geq 1$, we have the validity of (25). Therefore,

$$
I_{\gamma} \ll \tilde{\mathcal{A}}_{\gamma}^{q}\|f\|_{p}^{q}, \quad \gamma=0, \ldots, n-1
$$

where

$$
\tilde{\mathcal{A}}_{\gamma}=\max _{0 \leq l \leq \gamma} \sup _{k \geq 1}\left(\sum_{j=1}^{k}\left(d_{k, j}^{l, \gamma}\right)^{q} \Delta_{j}\right)^{\frac{1}{q}}\left(\sum_{i=k}^{\infty}\left(a_{i, k}^{(l)}\right)^{p^{\prime}} v_{i}^{-p^{\prime}}\right)^{\frac{1}{p^{\prime}}} .
$$

Using (14) and taking into account that $d_{i, j}^{l, n}$ is non-decreasing in $i$ and non-increasing in $j$, we find that

$$
\begin{gathered}
\sum_{j=1}^{k}\left(d_{k, j}^{l, \gamma}\right)^{q} \Delta_{j}=\sum_{m_{s+3} \leq k}\left(d_{k, m_{s+3}}^{l, \gamma}\right)^{q}\left(d_{m_{s+3}, m_{s+1}}^{\gamma, n}\right)^{q} \sum_{j=m_{s}+1}^{m_{s+1}} u_{j}^{q} \\
\ll \sum_{m_{s+3} \leq k} \sum_{i=m_{s}+1}^{m_{s+1}}\left(d_{k, i}^{l, n}\right)^{q} u_{i}^{q} \leq \sum_{i=1}^{k}\left(d_{k, i}^{l, n}\right)^{q} u_{i}^{q} .
\end{gathered}
$$


By combining (33), (34), and (35), we obtain that

$$
I_{\gamma} \ll \mathcal{A}_{n}^{q}\|f\|_{p, v}^{q}
$$

Thus, from (30), (31), and (36) it follows that

$$
\left\|A_{n}^{-} f\right\|_{q, u} \ll \mathcal{A}_{n}\|f\|_{p, v,} \quad f \geq 0,
$$

i.e., inequality (3) is valid and by Lemma 4.1, we obtain that

$$
C \ll \mathcal{A}_{n} \approx \mathcal{A}^{+} \approx \mathcal{A}^{-}
$$

2. If $N_{0} \neq N$, i.e., $\max N_{0}<\infty$ and $N_{0}=\left\{1,2, \ldots, s_{0}\right\}, s_{0} \geq 1$. Therefore, $m_{s_{0}}<\infty$ and $m_{s_{0}+1}=\infty$. We assume that $\sum_{s=k}^{n}=0$, if $k>n$ and $\sum_{s=k}^{n}=\sum_{s=1}^{n}$, if $k \leq 0$. We have two possible cases: $n_{s_{0}+1}<\infty$ and $n_{s_{0}+1}=\infty$. We consider these cases separately:

1) If $n_{s_{0}+1}<\infty$, then from (28) it follows that

$$
\begin{gathered}
\left\|A_{n}^{-} f\right\|_{q, u}^{q}=\sum_{s \in N_{0}} \sum_{j=m_{s}+1}^{m_{s+1}}\left(A_{n}^{-} f\right)_{j}^{q} u_{j}^{q}=\sum_{s=1}^{s_{0}} \sum_{j=m_{s}+1}^{m_{s+1}}\left(A_{n}^{-} f\right)_{j}^{q} u_{j}^{q} \\
=\sum_{s=1}^{s_{0}-3} \sum_{j=m_{s}+1}^{m_{s+1}}\left(A_{n}^{-} f\right)_{j}^{q} u_{j}^{q}+\sum_{s=s_{0}-2} \sum_{j=m_{s}+1}^{s_{s+1}-1}\left(A_{n}^{-} f\right)_{j}^{q} u_{j}^{q}+\sum_{j=m_{s_{0}}+1}^{\infty}\left(A_{n}^{-} f\right)_{j}^{q} u_{j}^{q}=J_{1}+J_{2}+J_{3} .
\end{gathered}
$$

If $J_{1} \neq 0$ then for $s_{0}>3$, we estimate $J_{1}$ using (29) and the previous proof for the case $N_{0}=\mathbb{N}$ as in estimate $I_{\gamma}$. Hence we get

$$
J_{1} \ll \mathcal{A}_{n}^{q}\|f\|_{p, v}^{q}
$$

If $J_{2} \neq 0$ then by using (27) and applying Hölder's and Jensen's inequalities, we obtain the following estimate

$$
\begin{aligned}
& J_{2}=\sum_{s=s_{0}-2}^{s_{0}-1} \sum_{j=m_{s}+1}^{m_{s+1}}\left(A_{n}^{-} f\right)_{j}^{q} u_{j}^{q}<\sum_{s=s_{0}-2}^{s_{0}-1} \sum_{j=m_{s}+1}^{m_{s+1}}(h+1)^{\left(-n_{s+1}+1\right) q} u_{j}^{q} \\
&=(h+1)^{q} \sum_{s=s_{0}-2}^{s_{0}-1}(h+1)^{-n_{s+1} q} \sum_{j=m_{s}+1}^{m_{s+1}} u_{j}^{q} \ll \sum_{s=s_{0}-2}^{s_{0}-1}\left(A_{n}^{-} f\right)_{m_{s+1}}^{q} \sum_{j=m_{s}+1}^{m_{s+1}} u_{j}^{q} \\
&= \sum_{s=s_{0}-2}^{s_{0}-1}\left(\sum_{i=m_{s+1}}^{\infty} a_{i, m_{s+1}}^{(n)} f_{i}\right)^{q} \sum_{J=m_{s}+1}^{m_{s+1}} u_{j}^{q} \\
& \leq \sum_{s=s_{0}-2}\left[\left(\sum_{i=m_{s+1}}^{\infty}\left(a_{i, m_{s+1}}^{(n)}\right)^{p^{\prime}} v_{i}^{-p^{\prime}}\right)^{\frac{1}{p^{\prime}}}\left(\sum_{j=m_{s}+1}^{m_{s+1}} u_{j}^{q}\right)^{\frac{1}{q}}\right]^{q}\left(\sum_{j=m_{s+1}}^{\infty}\left|v_{i} f_{i}\right|^{p}\right)^{\frac{q}{p}} \\
& \leq {\left[\sup _{k \geq 1}\left(\sum_{i=k}^{\infty}\left(a_{i, k}^{(n)}\right)^{p^{\prime}} v_{i}^{-p^{\prime}}\right)^{\frac{1}{p^{\prime}}}\left(\sum_{j=1}^{k} u_{j}^{q}\right)^{\frac{1}{q}}\right]^{q}\left(\sum_{s=s_{0}-2}^{s_{0}-1} \sum_{j=m_{s+1}}^{\infty}\left|v_{i} f_{i}\right|^{p}\right)^{\frac{q}{p}} } \\
& \leq 2 \mathcal{A}_{n}^{q}\|f\|_{p, v}^{q} \ll \mathcal{A}_{n}^{q}|| f \|_{p, v}^{q} .
\end{aligned}
$$


Using (27) and applying Hölder's inequality we estimate $J_{3}$ in the following way.

$$
\begin{gathered}
J_{3}=\sum_{j=m_{s_{0}}+1}^{\infty}\left(A_{n}^{-} f\right)_{j}^{q} u_{j}^{q} \leq \sup _{t \geq m_{s_{0}}+1} \sum_{j=m_{s_{0}}+1}^{t}\left(A_{n}^{-} f\right)_{j}^{q} u_{j}^{q} \\
\leq(h+1)^{q} \sup _{t \geq m_{s_{0}+1}}(h+1)^{-q n_{s_{0}+1}} \sum_{j=m_{s_{0}}+1}^{t} u_{j}^{q} \\
\ll \sup _{t \geq m_{s_{0}+1}}\left(A_{n}^{-} f\right)_{t}^{q} \sum_{j=m_{s_{0}}+1}^{t} u_{j}^{q}=\sup _{t \geq m_{s_{0}}+1}\left(\sum_{i=t}^{\infty} a_{i, t}^{(n)} f_{i}\right)^{q} \sum_{j=m_{s_{0}}+1}^{t} u_{j}^{q} \\
\leq \sup _{t \geq m_{s_{0}+1}}\left[\left(\sum_{i=t}^{\infty}\left(a_{i, t}^{(n)}\right)^{p^{\prime}} v_{i}^{-p^{\prime}}\right)^{\frac{1}{p^{\prime}}}\left(\sum_{j=m_{s_{0}}+1}^{t} u_{j}^{q}\right)^{\frac{1}{q}}\right]^{q}\|f\|_{p, v}^{q} \leq \mathcal{A}_{n}^{q}\|f\|_{p, v}^{q} .
\end{gathered}
$$

By (39), (40), (41), and (42) we obtain (37) and, consequently (38).

2) If $n_{s_{0}+1}=\infty$, which means that $k_{m_{s_{0}+1}}=\infty$, then by the definition of $m_{s_{0}+1}$ we have $T_{j}=\emptyset$, if $j \geq m_{s_{0}}+1$, i.e., $\left(A_{n}^{-} f\right)_{j}=0$, if $j \geq m_{s_{0}}+1$. and $T_{j}=\emptyset$, if $j \geq m_{s_{0}}+1$, i.e., $\left(A_{n}^{-} f\right)_{j}=0$, if $j \geq m_{s_{0}}+1$. By the assumption that $\left(A_{n}^{-} f\right)_{1} \neq 0$ it follows that $\mathrm{s}_{0}>1$. Therefore, $m_{2}<\infty$ and $s_{0} \geq 2$. Thus by (28) we have

$$
\begin{gathered}
\left\|A_{n}^{-} f\right\|_{q, u}^{q}=\sum_{s \in N_{0}} \sum_{j=m_{s}+1}^{m_{s+1}}\left(A_{n}^{-} f\right)_{j}^{q} u_{j}^{q} \\
=\sum_{s=1}^{s_{0}-3} \sum_{j=m_{s}+1}^{m_{s+1}}\left(A_{n}^{-} f\right)_{j}^{q} u_{j}^{q}+\sum_{s=s_{0}-2}^{s_{0}-1} \sum_{j=m_{s}+1}^{m_{s+1}}\left(A_{n}^{-} f\right)_{j}^{q} u_{j}^{q}=J^{\prime}{ }_{1}+J^{\prime}{ }_{2} .
\end{gathered}
$$

By estimating $J_{1}^{\prime}$ and $J_{2}^{\prime}$ as $J_{1}$ and $J_{2}$, respectively, from (43) we obtain (37) and, consequently (38). Therefore, we see that inequality (25) holds for $m=n$ and the estimate (24) is valid. This means that inequality (25) holds for all $m \geq 0$ with the estimate (24), which together with (23) gives $C \approx \mathcal{A}_{n}$. The proof is complete.

\section{Compactness of the matrix operators}

Theorem 5.1. Let $1<p \leq q<\infty$. Let the matrix $\left(a_{i, j}\right)$ of (1) belong to the class $\mathcal{O}_{n}^{+}, n \geq 0$. Then the operator defined by (1) is compact from $l_{p, v}$ into $l_{q, u}$ if and only if one of the following conditions holds

$$
\begin{aligned}
& \lim _{k \rightarrow \infty}\left(\mathcal{B}_{p, q}^{+}\right)_{k}=0, \\
& \lim _{k \rightarrow \infty}\left(\mathcal{B}_{p, q}^{-}\right)_{k}=0 .
\end{aligned}
$$

Theorem 5.2. Let $1<p \leq q<\infty$. Let the matrix $\left(a_{i, j}\right)$ of (2) belong to the class $\mathcal{O}_{m}^{-}, m \geq 0$. Then the operator defined by (2) is compact from $l_{p, v}$ into $l_{q, u}$ if and only if one of the following conditions holds

$$
\lim _{k \rightarrow \infty}\left(\mathcal{A}_{p, q}^{+}\right)_{k}=0,
$$




$$
\lim _{k \rightarrow \infty}\left(\mathcal{A}_{p, q}^{-}\right)_{k}=0
$$

Now we give the proof of compactness for the class $\mathcal{O}_{n}^{+}, n \geq 0$.

PROOF OF THEOREM 5.1. For the proof of Theorem 5.1, we need the following equivalence

$$
\left(\mathcal{B}_{p, q}^{+}\right)_{k} \approx\left(\mathcal{B}_{n}\right)_{k} \equiv \max _{0 \leq \gamma \leq n}\left(\mathcal{B}_{\gamma, n}\right)_{k} \approx\left(\mathcal{B}_{p, q}^{-}\right)_{k}
$$

where

$$
\left(B_{\gamma, n}\right)_{k}=\left(\sum_{i=k}^{\infty}\left(b_{i, k}^{n, \gamma}\right)^{q} u_{i}^{q}\right)^{\frac{1}{q}}\left(\sum_{j=1}^{k}\left(a_{k, j}^{(\gamma)}\right)^{p^{\prime}} v_{j}^{-p^{\prime}}\right)^{\frac{1}{p^{\prime}}} .
$$

The equivalence directly follows from (10).

Necessity. Suppose that the matrix of operator (1) belongs to the class $\mathcal{O}_{n}^{+}, n \geq 0$. Let the operator (1) be compact from $l_{p, v}$ into $l_{q, u}$.

For $r \geq 1$, we introduce the following sequence:

$$
\varphi_{r}=\left\{\varphi_{r, j}\right\}_{j=1}^{\infty}: \quad \varphi_{r, j}=\frac{f_{r, j}}{\left\|f_{r}\right\|_{p, v}},
$$

where $f_{r}=\left\{f_{r, j}\right\}_{j=1}^{\infty}: \quad f_{r, j}= \begin{cases}\left(a_{r, j}^{(\gamma)}\right)^{p^{\prime}-1} v_{j}^{-p^{\prime}}, & 1 \leq j \leq r, \\ 0, & j>r .\end{cases}$

It is obvious that $\varphi_{r p, v}=1$. Since the operator (1) is compact from $l_{p, v}$ into $l_{q, u}$, the set $\left\{u A^{+} \varphi,\|\varphi\|_{p, v}=1\right\}$ is precompact in $l_{q}$. Hence from criteria on precompactness of the sets in $l_{p}$ (see Theorem $\mathrm{B}$ ) we conclude that

$$
\lim _{r \rightarrow \infty} \sup _{\|\varphi\|_{p, v}=1}\left(\sum_{i=r}^{\infty} u_{i}^{q}\left(A^{+} \varphi\right)_{i}^{q}\right)^{\frac{1}{q}}=0 .
$$

Moreover, by using (9) we have that

$$
\begin{gathered}
\sup _{\|\varphi\|_{p, v}=1}\left(\sum_{i=r}^{\infty} u_{i}^{q}\left(A^{+} \varphi\right)_{i}^{q}\right)^{\frac{1}{q}} \geq\left(\sum_{i=r}^{\infty} u_{i}^{q}\left(A^{+} \varphi_{r}\right)_{i}^{q}\right)^{\frac{1}{q}} \\
=\left(\sum_{i=r}^{\infty} u_{i}^{q}\left(\sum_{j=1}^{i} a_{i, j}^{(n)} \frac{f_{r, j}}{\left\|f_{r}\right\|_{p, v}}\right)^{q}\right)^{\frac{1}{q}} \geq\left(\sum_{i=r}^{\infty} u_{i}^{q}\left(\sum_{j=1}^{r} a_{i, j}^{(n)} \frac{f_{r, j}}{\left\|f_{r}\right\|_{p, v}}\right)^{q}\right)^{\frac{1}{q}} \\
=\left(\sum_{i=r}^{\infty} u_{i}^{q}\left(\sum_{j=1}^{r} a_{i, j}^{(n)}\left(a_{r, j}^{(\gamma)}\right)^{p^{\prime}-1} v_{j}^{-p^{\prime}}\right)^{q}\right)^{\frac{1}{q}}\left(\sum_{j=1}^{r}\left(a_{r, j}^{(\gamma)}\right)^{p^{\prime}} v_{j}^{-p^{\prime}}\right)^{-\frac{1}{p}} \\
\geq\left(\sum_{i=r}^{\infty} u_{i}^{q}\left(b_{i, r}^{n, \gamma}\right)^{q}\right)^{\frac{1}{q}}\left(\sum_{j=1}^{r}\left(a_{r, j}^{(\gamma)}\right)^{p^{\prime}} v_{j}^{-p^{\prime}}\right)^{\frac{1}{p^{\prime}}}=\left(\mathcal{B}_{\gamma, n}\right)_{r} .
\end{gathered}
$$


Since inequality (50) hold for all $\gamma=0,1, \ldots n$ and from the validity of (49) we obtain

$$
\lim _{r \rightarrow \infty}\left(\mathcal{B}_{n}\right)_{r}=0
$$

The proof of the necessity is complete.

Sufficiency. Let the matrix of operator (1) belong to the class $\mathcal{O}_{n}^{+}, n \geq 0$. Assume that at least one of the conditions (44) and (45) is valid. Then, by Theorem 4.1, the operator (1) is bounded from $l_{p, v}$ into $l_{q, u}$. Consequently, the set $\left\{u A^{+} f,\|f\|_{p, v} \leq 1\right\}$ is bounded in $l_{q}$. Let us show that this set is precompact in $l_{q}$. By the criteria on precompactness of the sets in $l_{q}$ (see Theorem B), the bounded set $\left\{u A^{+} f,\|f\|_{p, v} \leq 1\right\}$ is compact in $l_{q}$, if

$$
\lim _{r \rightarrow \infty} \sup _{\|f\|_{p, v} \leq 1}\left(\sum_{i=r}^{\infty} u_{i}^{q}\left|\left(A^{+} f\right)_{i}\right|^{q}\right)^{\frac{1}{q}}=0 .
$$

For $r>1$ we assume that $\tilde{u}=\left\{\tilde{u}_{i}\right\}_{i=1}^{\infty}: \quad \tilde{u}_{i}=\left\{\begin{array}{l}0, \quad 1 \leq i \leq r-1 \\ u_{i}, r \leq i .\end{array}\right.$

Then, by Theorem 4.1, we have that

$$
\sup _{\|f\|_{p, v} \leq 1}\left(\sum_{i=r}^{\infty} u_{i}^{q}\left|\left(A^{+} f\right)_{i}\right|^{q}\right)^{\frac{1}{q}}=\sup _{\|f\|_{p, v} \leq 1}\left(\sum_{i=1}^{\infty} \tilde{u}_{i}^{q}\left|\left(A^{+} f\right)_{i}\right|^{q}\right)^{\frac{1}{q}} \ll \tilde{B}_{n}(r),
$$

where

$$
\tilde{B}_{n}(r)=\sup _{k \geq 1} \max _{0 \leq \gamma \leq n}\left(\sum_{i=k}^{\infty}\left(b_{i, k}^{n, \gamma}\right)^{q} \tilde{u}_{i}^{q}\right)^{\frac{1}{q}}\left(\sum_{j=1}^{k}\left(a_{k, j}^{(\gamma)}\right)^{p^{\prime}} v_{j}^{-p^{\prime}}\right)^{\frac{1}{p^{\prime}}} .
$$

Since $\tilde{u}_{i}=0$ when $1 \leq i \leq r-1$ we have

$$
\tilde{B}_{n}(r)=\sup _{k \geq r} \max _{0 \leq \gamma \leq n}\left(\sum_{i=k}^{\infty}\left(b_{i, k}^{n, \gamma}\right)^{q} u_{i}^{q}\right)^{\frac{1}{q}}\left(\sum_{j=1}^{k}\left(a_{k_{j} i}^{(\gamma)}\right)^{p^{\prime}} v_{j}^{-p^{\prime}}\right)^{\frac{1}{p^{\prime}}}=\sup _{k \geq r}\left(B_{n}\right)_{k},
$$

By (44), (45), (48), and (53) we deduce

$$
\lim _{r \rightarrow \infty} \tilde{B}_{n}(r)=\lim _{r \rightarrow \infty} \sup _{k \geq r}\left(B_{n}\right)_{k}=\lim _{r \rightarrow \infty}\left(B_{n}\right)_{r}=0 .
$$

Hence, by using (52) we obtain (51) and the proof is complete.

Theorem 5.2 is proven in a similar way.

Now on the base of Theorems 4.1, 4.2, 5.1, and 5.2 we prove our main results.

\section{Proof of Theorem 3.1}

(i) If the matrix of (1) belong to $\mathcal{O}_{m}^{+}, m \geq 0$.then the statement (i) of Theorem 3.1 directly follows from Theorem 4.1. Suppose that the matrix $\left(a_{i, j}\right)=\left(a_{i, j}^{(m)}\right)$ of $(1)$ belongs to $\mathcal{O}_{m}^{-}, m \geq 0$. It is known that the boundedness of operator (1) from $l_{p, v}$ into $l_{q, u}$ is equivalent to the boundedness of conjugate operator from $l_{q^{\prime}, u^{-1}}$ into $l_{p^{\prime}, v^{-1}}$, which coincides with operator (2). From the condition that $1<p \leq q<\infty$ it follows 
that $1<q^{\prime} \leq p^{\prime}<\infty$. Then by Theorem 4.2 and the identities $\left(\mathcal{A}_{q^{\prime}, p^{\prime}}^{+}\right)_{k}=\left(\mathcal{B}_{p, q}^{+}\right)_{k}$ and $\left(\mathcal{A}_{q^{\prime}, p^{\prime}}^{-}\right)_{k}=\left(\mathcal{B}_{p, q}^{-}\right)_{k}$, the boundedness of the operator defined by (2) from $l_{q^{\prime}, u^{-1}}$ into $l_{p^{\prime}, v^{-1}}$ is equivalent to the conditions of the statement (i) of Theorem 3.1. Hence the statement (i) of Theorem 3.1 is also valid in the case when the matrix of (1) belongs to $\mathcal{O}_{m}^{-}, m \geq 0$. The proof of the statement (i) of Theorem 3.1 is complete.

(ii) Let the matrix of (1) belong to $\mathcal{O}_{m}^{+}, m \geq 0$. Then the statement (ii) of Theorem 3.1 follows from Theorem 5.1. If the matrix $\left(a_{i, j}\right)=\left(a_{i, j}^{(m)}\right)$ of (1) belongs to $\mathcal{O}_{m}^{-}, m \geq 0$, then as stated above based on Theorem 5.2 it follows the statement (ii) of Theorem 3.1.

The proof of Theorem 3.1 is complete.

The proof of Theorem 3.2 can be carried out by the same method as in the proof of Theorem 3.1.

REMARK 4. If we consider operator defined by (1) and operator of the following form

$$
\left(\Sigma^{+} g\right)_{i}=\sum_{j=1}^{i} \sigma_{i, j} g_{j,} \quad i \geq 1 .
$$

Then

$$
\begin{gathered}
\left(A^{+} \circ \Sigma^{+}\right)(g)_{i} \equiv\left(A^{+}\left(\Sigma^{+} g\right)\right)_{i}=\sum_{j=1}^{i} a_{i, j} \sum_{k=1}^{j} \sigma_{j, k} g_{k} \\
=\sum_{k=1}^{i}\left(\sum_{j=k}^{i} a_{i, j} \sigma_{j, k}\right) g_{k}=\sum_{k=1}^{i} w_{i, k} g_{k} .
\end{gathered}
$$

Therefore if $\left(a_{i, j}\right) \in \mathcal{O}_{n^{\prime}}^{+}\left(\sigma_{j, k}\right) \in \mathcal{O}_{m^{\prime}}^{+}$then according to Lemma 2.1 the matrix $\left(w_{i, k}\right)$ of the operator $A^{+} \circ \Sigma^{+}$belongs to the class $\mathcal{O}_{m+n+1}^{+}$.

In general case, if matrices $\left(a_{i, j}^{k}\right)$ of operators $\left(A_{k}^{+} f\right)_{j}=\sum_{j=1}^{i} a_{i, j}^{k} f_{j}$ belong to the classes $\mathcal{O}_{m_{k}}^{+}, k=1, \ldots, n$, then the matrix of operator $\mathbb{A}_{n}^{+} \equiv A_{1}^{+} \circ A_{2}^{+} \circ \cdots \circ A_{n}^{+}$belongs to the class $\mathcal{O}_{m}^{+}$, where $m=\sum_{k=1}^{n} m_{k}+n-1$. So according to Theorem 3.1 we obtain criteria of boundedness and compactness of the matrix operator $\mathbb{A}_{n}^{+}$from the weighted $l_{p, v}$ space into the weighted $l_{q, u}$ space, $1<p \leq q<\infty$

Similarly, if matrices $\left(a_{i, j}^{k}\right)$ of operators $\left(A_{k}^{-} g\right)_{j}=\sum_{i=j}^{\infty} a_{i, j}^{k} g_{i}$ belong to the classes $\mathcal{O}_{m_{k}}^{-}, k$ $=1, \ldots, n$, then based on Lemma 2.2, Theorem 3.2 gives necessary and sufficient conditions for boundedness and compactness of operator $\mathbb{A}_{n}^{-}=A_{1}^{-} \circ A_{2}^{-} \circ \cdots \circ A_{n}^{-}$from $l_{p, v}$ into $l_{q, u}, 1<p \leq q<\infty$. 


\section{Competing interests}

The authors declare that they have no competing interests.

Received: 15 September 2011 Accepted: 2 March 2012 Published: 2 March 2012

\section{References}

1. Kufner, A, Maligranda, L, Persson, LE: The Hardy Inequality. About its History and Some Related Results. Vydavatelsky Servis Publishing House, Pilsen. (2007)

2. Kokilashvili, V, Meskhi, A, Persson, LE: Weighted norm inequalities for integral trans-forms with product kernals. Nova Science Publishers, Inc, New York. (2010)

3. Kufner, A, Persson, LE: Weighted Inequalities of Hardy Type. World Scientic Publishing Co, Singapore. (2003)

4. Oinarov, R, Okpoti, CA, Persson, LE: Weighted inequalities of Hardy type for matrix operators: the case $q<p$. Math Inequal Appl. 10, 843-861 (2007)

5. Oinarov, R, Shalgynbaeva, SK: Weighted additive estimate of a class of matrix operators. Izvestiya NAN RK, serial PhysMat (in Russian). 1, 39-49 (2004)

6. Oinarov, R, Persson, LE, Temirkhanova, AM: Weighted inequalities for a class of matrix operators: the case $p \leq q$. Math Inequal Appl. 12, 891-903 (2009)

7. Temirkhanova, AM, Taspaganbetova, ZA: Boundedness and compactness criteria of a certain class of matrix operators. Math J. 11, 125-139 (2011)

8. Temirkhanova, AM, Taspaganbetova, ZA: Criteria on Boundedness of matrix operators in weighted spaces of sequences and their applications. Annals Funct Anal. 1, 114-127 (2011)

9. Okpoti, CA, Persson, LE, Wedestig, A: Scales of weight characterizations for the discrete Hardy and Carleman type inequalities. In: Pavel Drabek, Jiri Rakosnik (eds.) Proc Conf "Function spaces, Differential operators and Nonlinear Analysis"236-258 (2005). FSDONA 2004 (Milovy, May 28-Jun 2, 2004), Math. Inst. Acad. Sci., Chech Republic, Prague

10. Birman, MSh, Vilenkin, NYa, Gorin, EA, Zabreiko, PP, Iohvidov, IS, Kadec, MI, Kostiuchenko, AG, Krasnosel'skii, MA, Krein, SG, Mityagin, BS, Petunin, IU, Rutickii, YaB, Semenov, EM, Sobolev, VI, Stecenko, VYa, Faddeev, LD, Citlanadze, ES: Reference book Mathematical Library. Functional Analysis. In: Krein SG (ed.) Chapter I, Concepts of analysis. Nauka, Moscow (1972). (in Russian)

doi:10.1186/1029-242X-2012-53

Cite this article as: Oinarov and Taspaganbetova: Criteria of boundedness and compactness of a class of matrix operators. Journal of Inequalities and Applications 2012 2012:53.

\section{Submit your manuscript to a SpringerOpen ${ }^{\odot}$ journal and benefit from:}

- Convenient online submission

Rigorous peer review

- Immediate publication on acceptance

- Open access: articles freely available online

- High visibility within the field

- Retaining the copyright to your article

Submit your next manuscript at $\gg$ springeropen.com 